\title{
Zosteriform Cutaneous Leishmaniasis of the Elbow
}

\section{Salem Bouomrani ${ }^{1,2, *}$, Safa Trabelsi ${ }^{1,2}$}

${ }^{1}$ Department of Internal medicine, Military Hospital of Gabes, Gabes 6000, Tunisia

${ }^{2}$ Sfax Faculty of Medicine, University of Sfax, Sfax 3029, Tunisia.

*Corresponding Author: Dr. Salem Bouomrani, Department of Internal medicine, Military Hospital of Gabes, Gabes 6000, Tunisia.

\section{Abstract}

Clinical features of cutaneous leishmaniasis (CL) are characterized by a very significant polymorphism making its diagnosis a real challenge for healthcare professionals.

Herpes-zoster-like or zosteriform leishmaniasis is exceptional and often misdiagnosed atypical/unusual presentation of CL. Its prevalence is estimated at 0.14-0.17\% of all CL and $2.4 \%$ of atypical $C L$, and is often reported as sporadic clinical cases.

We present an original case of zosteriform CL at the elbow, in a young immunocompetent Tunisian woman.

Keywords: zosteriform leishmaniasis, cutaneous leishmaniasis, atypical.

\section{INTRODUCTION}

Cutaneous leishmaniasis (CL) is a parasitosis still frequent worldwide, and represents an important public-health problem in many countries [1,2]. Indeed, according to the world health organization (WHO), more than 350 million people are currently at risk for this infection [2].

Clinical features of this infection are characterized by a very significant polymorphism making its diagnosis a real challenge for healthcare professionals [1-3].

The authors described 17 presentations qualified as atypical CL, among them the most frequent are: sporotrichoid, acute paronychial, chancriform, and lupoid leishmaniasis [1-3]. Other forms are much rarer: palmoplantar, lid, psoriasiform, annular, whitlow, erysipeloid, and zosteriform leishmaniasis [1-3].

Herpes-zoster-like or zosteriform leishmaniasis is exceptional and often misdiagnosed unusual presentation of CL.

We present an original case of zosteriform CL in a young immunocompetent Tunisian woman.

\section{CASE REPORT}

Tunisian woman, 28-years-old, with no pathological medical history, was referred to our department for suspicion of herpes-zoster. The diagnosis of herpeszoster of the right elbow was mentioned by his family doctor, but no clinical improvement was observed after ten days of treatment with aciclovir.

The somatic examination showed multiple papules and pseudo-vesicles in a lineal pattern (Figs.1 \& 2) around a main ulcero-crusted lesion (Fig.3) in the right elbow without other anomalies. The basic biology tests were within normal limits.

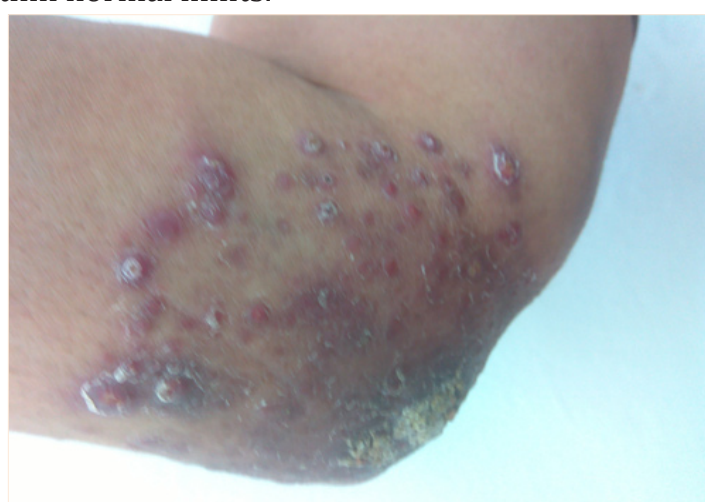

Figure 1. Right elbow in flexion: multiple papules and pseudo-vesicles in a lineal pattern. 


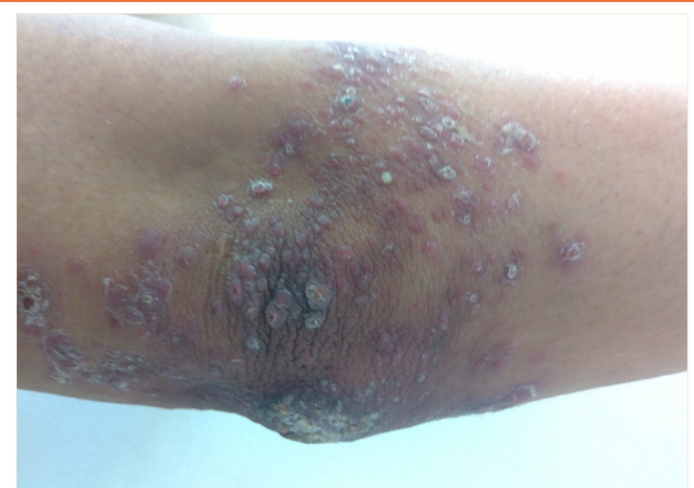

Figure 2. Right elbow in extension: multiple papules and pseudo-vesicles in a lineal pattern

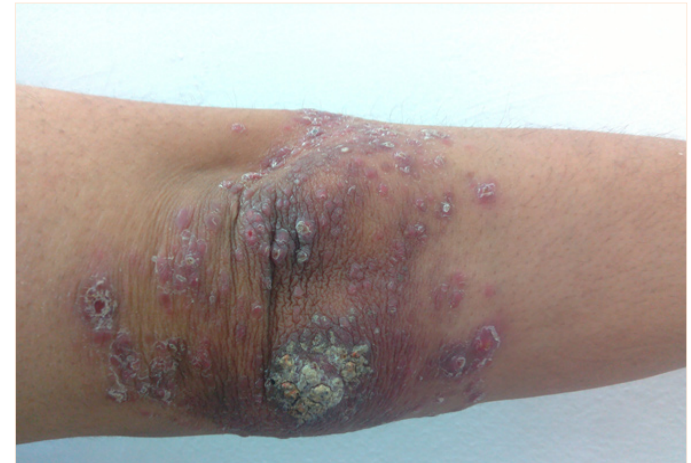

Figure 3. Right elbow in extension: the main ulcerocrusted lesion with multiple satellite lesions.

The diagnosis of CL was confirmed by the detection of Leishmania amastigotes in smear from the ulcerocrusted lesion.

The patient was treated with intramuscular meglumine antimoniate at the dose of $60 \mathrm{mg} / \mathrm{kg} /$ day for two weeks with favorable evolution: progressive disappearance of the satellite lesions from the tenth day of treatment (Figs.4 \& 5) and their complete disappearance and healing of the main lesion on the fifteenth day (Fig.6).

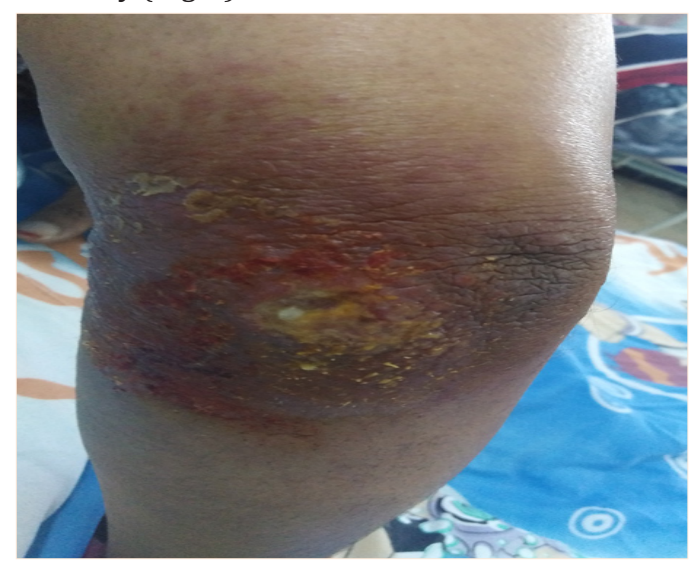

Figure 4. Lesions after one week treatment.

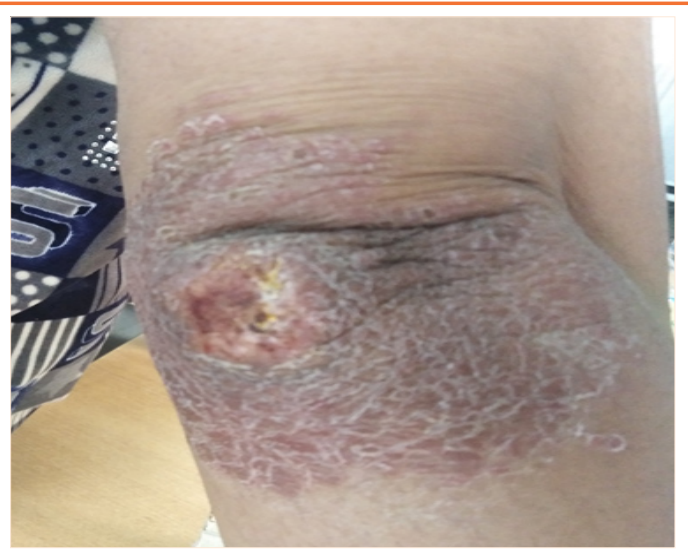

Figure 5. Lesions after ten days treatment.

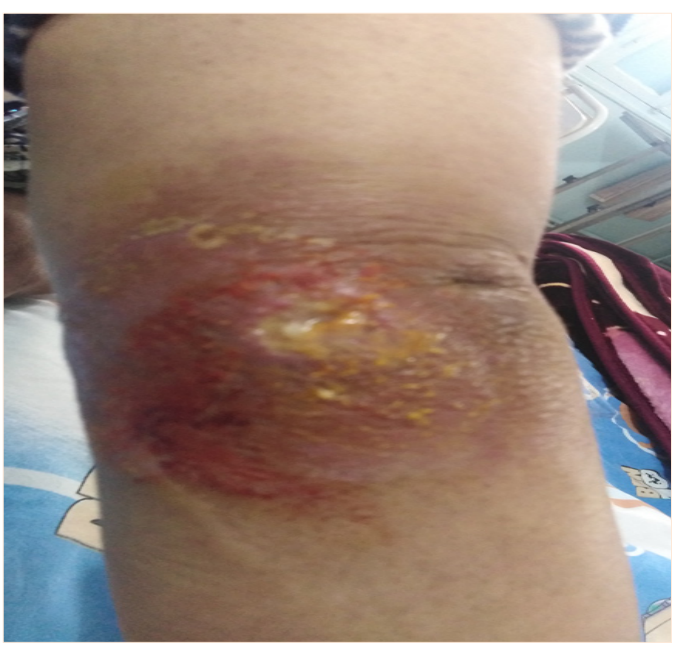

Figure 6. Lesion after two weeks treatment.

\section{Discussion}

The overall frequency of atypical presentations of CL is estimated at $2.5 \%[3,4]$. The zosteriform one is exceptional $[1,3,5]$, and only anecdotally reported in the literature [5].

In Bari $\mathrm{AU}$ et al, series of 718 patients with CL, only one patient had zosteriform leishmaniasis $(0.14 \%$ of all CL and $2.4 \%$ of atypical CL), and in the large series of Raja KM et al, of $1709 \mathrm{CL}$, only three had zosteriform presentation $(0.17 \%)$ [4].

Clinically, zosteriform leishmaniasis is characterized by linear arrangement of satellite papules and pseudovesicles in a lineal pattern around the main lesion $[4,6]$. Extension of lesions can affect one or more dermatomes $[4,6]$.

The exact pathophysiology of this presentation is not well known; the alteration of the host's immune response as well as an atypical strain of parasites in the lesions are mentioned by most authors $[1,5,6]$. 
The diagnosis of CL in front of this form may be overlooked even in endemic areas [1,2], and patient can mistakenly be treated for herpes-zoster in several occasion as in the observation of Omidian $\mathrm{M}$ et al [1]. Dermoscopy can be very helpful for the diagnosis of this atypical variant of CL [5].

This form of CL can resist to conventional treatment [1]; in this case association of meglumine antimoniate, allopurinol, and cryotherapy can be proposed [1].

\section{CONCLUSION}

As rare as it is, this unusual clinical form of CL deserves to be well known by healthcare professionals, particularly those in primary care. This diagnosis should be discussed in front of any herpes-zosterlike lesion that does not improve under appropriate antiviral therapy. Our observation is distinguished by its location at the elbow which, to our knowledge, has not been reported before.

\section{Conflicts of Interest: None}

\section{REFERENCES}

1. Omidian M, Mapar MA. Chronic zosteriform cutaneous leishmaniasis. Indian J Dermatol Venereol Leprol. 2006;72(1):41-2.
2. Remadi L, Haouas N, Chaara D, Slama D, Chargui $\mathrm{N}$, Dabghi R, et al. Clinical Presentation of Cutaneous Leishmaniasis caused by Leishmania major. Dermatology. 2016;232(6):752-759.

3. Bari A, Rahman SB. Many faces of cutaneous leishmaniasis. Indian J Dermatol Venereol Leprol 2008;74:23-7

4. Raja KM, Khan AA, Hameed A, Rahman SB. Unusual clinical variants of cutaneous leishmaniasis in Pakistan. Br J Dermatol. 1998;139(1):111-3.

5. Ramot Y, Nanova K, Alper-Pinus R, Zlotogorski A. Zosteriform cutaneous leishmaniasis diagnosed with the help of dermoscopy. Dermatol Pract Concept. 2014;4(3):55-7.

6. Morales CA, Palacio J, Rodríguez G, Camargo YC. Zosteriform cutaneous leishmaniasis due to Leishmania ( Viannia) panamensis and Leishmania (Viannia ) braziliensis: report of three cases. Biomedica. 2014;34(3):340-4.

Citation: Salem Bouomrani, Safa Trabelsi. Zosteriform Cutaneous Leishmaniasis of the Elbow. Archives of Dermatology and Skin Care. 2020; 3(2): $01-03$

Copyright: (C) 2020 Salem Bouomrani, Safa Trabelsi. This is an open access article distributed under the Creative Commons Attribution License, which permits unrestricted use, distribution, and reproduction in any medium, provided the original work is properly cited. 